\title{
Responsabilidad social sobre contaminación acústica en los estudiantes de Ingeniería Industrial, Sistemas e Informática.
}

Social responsibility about acoustic contamination in the students of the ability of Industrial Engineering, Systems and Computer Science.

Alfredo Edgar López Jiménez', William Fredy López Jiménez', Elver Stalin Ticliahuanca Calderón`

\section{RESUMEN}

Objetivo: Analizar la relación entre el nivel de conocimientos en contaminación acústica y la protección del medio ambiente en los estudiantes de ingeniería. Métodos: Se utilizó el modelo del diseño de investigación descriptivo correlacional. La población del estudio fue de 1200 estudiantes de la Facultad de Ingeniería Industrial, Sistemas e Informática, del que se calculó un tamaño de muestra final de 234 estudiantes. Resultados: Los resultados indican que tienen niveles intermedios de conocimientos, representando a la mayoría con un porcentaje del 59,6\%. Sobre la variable conciencia en protección del medio ambiente, los resultados son relativamente Altos, 52,1\%. Conclusiones: Se comprueba que el nivel de conocimientos en contaminación acústica si se relaciona con la protección del medio ambiente en los estudiantes de ingeniería.

Palabras clave: Responsabilidad, responsabilidad social, contaminación, contaminación acústica.

\section{ABSTRACT}

Objective: To analyze the relationship between the level of knowledge in noise pollution and the protection of the environment in engineering students. Methods: The correlational descriptive research design model was used. The study population was 1200 students from the Faculty of Industrial Engineering, Systems and Informatics, from which a final sample size of 234 students was calculated. Results: The results indicate that they have intermediate levels of Knowledge, representing the majority with a percentage of $59,6 \%$. Regarding the variable environmental protection awareness, the results are relatively high, $52,1 \%$. Conclusions: It is verified that the level of knowledge in noise pollution is related to the protection of the environment in engineering students.

Keyword: Responsibility, social responsibility, contamination, acoustic contamination.

\footnotetext{
${ }^{1}$ Universidad Nacional José Faustino Sánchez Carrión, Huacho, Perú.
} 


\section{INTRODUCCIÓN}

En nuestro país la contaminación del medio ambiente, es una preocupación seria, por ello nuestros ciudadanos comienzan a tomar conciencia del cuidado ó protección del medio ambiente. La toma de conciencia se viene promoviendo desde los menores de edad, y cuando lleguen a la adolescencia ya tienen que manifestar un compromiso definido.

La contaminación acústica o contaminación sonora, es uno de los factores que afectan la calidad de vida de los ciudadanos, muchos son perturbados en su tranquilidad. Se describe a la contaminación acústica o contaminación sonora, como el exceso de niveles de intensidad del sonido, que altera las condiciones normales del ambiente en una determinada zona. El término de "contaminación acústica" hace referencia al Ruido (entendido como sonido excesivo y molesto), provocado por las actividades humanas (tráfico, industrias, locales de ocio, aviones, barcos, entre otros.) que produce efectos nocivos fisiológicos y psicológicos para una persona o grupo de personas (efectos negativos sobre la salud auditiva, física y mental de los seres vivos) .

El ruido es una de las principales causas de preocupación entre la población de las ciudades, ya que incide en el nivel de calidad de vida y además puede provocar efectos nocivos sobre la salud, el comportamiento y actividades del hombre, y provoca efectos psicológicos y sociales. El incremento de los niveles de ruido ha crecido de forma desproporcionada en las últimas décadas y sólo en España se calcula que al menos 9 millones de personas soportan niveles medios de 65 decibelios $(\mathrm{db})$, siendo el segundo país, detrás de Japón, con mayor índice de población expuesta a altos niveles de contaminación acústica (Waste, 2017).

Los niveles de ruido siguen excediendo regularmente los valores límite en muchos sectores, como agricultura, construcción, ingeniería, alimentos y bebidas, industria, carpintería, fundiciones o entretenimiento (Facts, 2017).

Si bien el ruido no se acumula, traslada o mantiene en el tiempo como las otras contaminaciones, también puede causar grandes daños en la calidad de vida de las personas si no se controla bien o adecuadamente.

Las principales causas de la contaminación acústica son aquellas relacionadas con las actividades humanas como el transporte, la construcción de edificios, obras públicas y las industrias, entre otras (Facts, 2017).
Se ha dicho por organismos internacionales, que se corre el riesgo de una disminución importante en la capacidad auditiva, así como la posibilidad de trastornos que van desde lo psicológico (paranoia, perversión) hasta lo fisiológico por la excesiva exposición a la contaminación sónica (Facts, 2017).

Un informe de la Organización Mundial de la Salud (OMS), considera los $70 \mathrm{~dB}(\mathrm{a})$, como el límite superior permitido.

Según los Ecologistas en acción (2004) mencionan diferentes estudios sobre las consecuencias del ruido en las personas. La población expuesta a un nivel de ruido por encima de los 65 decibelios desarrolla a corto plazo un índice superior en un $20 \%$ de ataques cardíacos. (Estudio Cohort, presentación en Barcelona a cargo de Dieter Gottlob, de la Agencia Federal Alemana de Medio Ambiente Alemana). Los niños y los ancianos son más sensibles a los ruidos que perturban su sueño, aunque su reacción no es la misma: mientras los ancianos son más propensos a despertarse debido a la ligereza de su sueño, ambos grupos mostraron alteraciones vitales debido al ruido, aun durmiendo a pierna suelta: alteraciones del pulso, vasoconstricción, modificaciones en el electromiógrafo y en el encefalograma. (Experimento realizado por el Doctor Alain Muzet, del Centro de Estudios Bioclimáticos del CNRS, en Francia). Con niveles de ruido altos, la tendencia natural de la gente hacia la ayuda mutua disminuye o desaparece, reapareciendo en el momento en que se suprime la presión sonora. En experimentos de laboratorio con animales se demostró que en un ambiente con ruido superior a 110 decibelios (claxon de automóvil a un metro, sirena de ambulancia a la misma distancia, discoteca, concierto de rock, moto a escape libre, trueno), los procesos cancerosos aparecen y se desarrollan con mayor rapidez.

Los niños cuyos colegios lindan con zonas ruidosas (industrias, aeropuertos, carreteras con mucho tráfico), aprenden a leer más tarde, presentan mayor agresividad, fatiga, agitación, peleas y riñas frecuentes, mayor tendencia al aislamiento, y cierta dificultad de relación con los demás. EI CSIC afirma a este respecto que la contaminación acústica conlleva efectos negativos en las generaciones futuras, como deterioro del aprendizaje y del desarrollo humano.

Por ello, el objetivo de la presente investigación fue analizar la relación entre el nivel de conocimientos en contaminación acústica y la protección del medio ambiente en los estudiantes de ingeniería. 


\section{MATERIAL Y MÉTODOS}

La investigación se realizó en los ambientes de las aulas de la Facultad de Ingeniería Industrial, Sistemas e Informática de la Universidad Nacional José Faustino Sánchez Carrión. El tamaño de muestra calculado fue de 234 estudiantes matriculados, se utilizó el muestreo simple o aleatorio, el cual cumplió con la característica de ser probabilístico. Estos estudiantes fueron encuestados al azar en el interior de las aulas de la Facultad en estudio. El Instrumento utilizado ha sido un modelo de encuesta elaborado por los propios autores, con cuestionario para medir los conocimientos sobre la responsabilidad social sobre contaminación acústica; contaminación acústica de la industria, contaminación acústica del transporte, contaminación acústica del comercio y conciencia en protección del medio ambiente. Este instrumento fue evaluado con el alpha de cronbach. Al respecto Córdova (2018), manifiesta que la confiabilidad de un instrumento "es la capacidad que tiene un instrumento de hacer una medición real de una variable, así como la consistencia o estabilidad de la medición".

\section{RESULTADOS}

El coeficiente alpha de Cronbach resulto con un valor del 0,89, el cual lo ubica en una categoría de alta confiabilidad, así mismo tiene una validación de juicio de expertos del $90,0 \%$, que lo valida como muy bueno. En la Tabla 1 se muestran los resultados descriptivos de la investigación. En ella se consideraron los cuatro indicadores evaluados en el presente estudio.

Tabla 1. Resultados Descriptivos de la Investigación.

\begin{tabular}{|c|c|c|c|}
\hline Indicadores & Categoria & Frecuencia & Porcentaje \\
\hline \multirow{3}{*}{$\begin{array}{l}\text { 1.- Nivel de conocimientos sobre } \\
\text { contaminación acústica de la } \\
\text { industria. }\end{array}$} & Malo & 30 & $12,8 \%$ \\
\hline & Regular & 140 & $59,8 \%$ \\
\hline & Bueno & 64 & $27,4 \%$ \\
\hline \multirow{3}{*}{$\begin{array}{l}\text { 2. Nivel de conocimientos sobre } \\
\text { contaminación acústica del } \\
\text { transporte. }\end{array}$} & Malo & 20 & $8,5 \%$ \\
\hline & Regular & 94 & $40,2 \%$ \\
\hline & Bueno & 120 & $51,3 \%$ \\
\hline \multirow{3}{*}{$\begin{array}{l}\text { 3. Nivel de conocimientos sobre } \\
\text { contaminación acústica del } \\
\text { comercio. }\end{array}$} & Malo & 38 & $16,2 \%$ \\
\hline & Regular & 96 & $41,0 \%$ \\
\hline & Bueno & 100 & $42,7 \%$ \\
\hline \multirow{3}{*}{$\begin{array}{l}\text { 4. Conocimientos sobre } \\
\text { responsabilidad social en } \\
\text { contaminación acústica. }\end{array}$} & Malo & 11 & $4,7 \%$ \\
\hline & Regular & 121 & $51,7 \%$ \\
\hline & Bueno & 102 & $43,6 \%$ \\
\hline \multirow{3}{*}{$\begin{array}{l}\text { 5. Conciencia sobre protección del } \\
\text { medio ambiente }\end{array}$} & Malo & 12 & $5,1 \%$ \\
\hline & Regular & 100 & $42,7 \%$ \\
\hline & Bueno & 122 & $52,1 \%$ \\
\hline
\end{tabular}

En el nivel de conocimientos sobre contaminación acústica de la industria, se visualiza que el $27,4 \%$ de los estudiantes muestran una categoría bueno para el nivel de conocimientos sobre contaminación acústica de la industria, mientras que solo el 12,8\% se encontró en la categoría de malo. En el nivel de conocimientos sobre contaminación acústica del transporte, se observa que el $51,3 \%$ de estudiantes muestra un conocimiento bueno y el $40,2 \%$ de estudiantes muestra una categoría regular de conocimiento.

En el nivel de conocimientos sobre contaminación acústica del comercio, se visualiza un $42,7 \%$ de estudiantes cuyo conocimiento es bueno y un $41 \%$ se encuentra en la categoría regular. Estos resultados sugieren falta de compromiso social 
sobre responsabilidad social en contaminación acústica.

En la variable responsabilidad social sobre contaminación acústica se aprecia un $51,7 \%$ de estudiantes cuyo conocimiento es regular, mientras que un $43,6 \%$ pertenecen a la categoría bueno, mientras que en la variable conciencia sobre protección del medio ambiente se aprecia que el $52,1 \%$ de los estudiantes muestra una conciencia alta y el $42,7 \%$ tienen conciencia media.

Estos resultados nos sugieren que se debe de mantener este interés estudiantil para proteger el medio ambiente, y cuidar nuestro ecosistema.

En la Tabla 2 se muestran los resultados inferenciales de la investigación. Todos los factores de conocimientos evaluados en los estudiantes tuvieron una relación estadísticamente significativa $(p<0,05)$.

Tabla 2. Resultados Inferenciales de la Investigación.

\begin{tabular}{lccc}
\hline \multirow{2}{*}{$\begin{array}{c}\text { Contrastación de Factores } \\
\text { Conocimientos en: }\end{array}$} & \multicolumn{2}{c}{ Conciencia en protección del medio ambiente } \\
\cline { 2 - 4 } & $\begin{array}{c}\text { Valor Chi-cuadrado } \\
\text { de Pearson }\end{array}$ & Significancia & Interpretación \\
\hline Contaminación acústica de la industria & 13,959 & 0,007 & Altamente significativo \\
Contaminación acústica del transporte & 16,360 & 0,003 & Altamente significativo \\
Contaminación acústica del comercio & 18,209 & 0,001 & Altamente significativo \\
$\begin{array}{l}\text { Responsabilidad social en } \\
\text { contaminación acústica }\end{array}$ & 31,791 & 0,000 & Altamente significativo
\end{tabular}

\section{DISCUSIÓN}

De acuerdo con los resultados de nuestra investigación, se confirma que el nivel de conocimientos sobre responsabilidad social sobre contaminación acústica si se relaciona con la protección del medio ambiente en los estudiantes de la facultad de Ingeniería Industrial, Sistemas e Informática. Los conocimientos sobre la responsabilidad social de la contaminación acústica en los estudiantes de ingeniería, tiene los indicadores contaminación acústica de la industria, del transporte y el comercio. Estos conocimientos en promedio tienen la categoría de intermedio, lo cual indica que los estudiantes tienen limitaciones en sus reflexiones para el cuidado y protección del medio ambiente. Las mejoras de los conocimientos y capacidades de los estudiantes universitarios de ingeniería, para promover el cuidado del medio ambiente sería una buena oportunidad para la comunidad universitaria y así egresar profesionales con alto grado de sensibilidad en el cuidado y protección del medio ambiente.

La contaminación acústica, es uno de los factores que afectan el cuidado del medio ambiente, para la física «es una sensación producida en el oído por determinadas oscilaciones de la presión exterior. La sucesión de compresiones y enrarecimientos que provoca la onda acústica al desplazarse por el medio hace que la presión existente fluctúe en torno a su valor de equilibrio; estas variaciones de presión actúan sobre la membrana del oído y provocan en el tímpano vibraciones forzadas de idéntica frecuencia, originando la sensación de sonido» (Fernández, 2000). Como ha señalado este autor en el sonido aparecen dos conceptos esencialmente distintos, aunque íntimamente relacionados; por un lado, la onda sonora o ente físico capaz de producir la sensación de sonido y, por otro, la sonoridad o sensación subjetiva producida por ciertas variaciones de presión en el oído. Pero el ruido no sería considerado como tal si, no produjese un rechazo y efectos no deseados para el que lo sufre.

En cualquier caso, lo esencial de cualquier definición es que se trata de uno o diversos sonidos molestos que pueden producir efectos fisiológicos, psicológicos y sociales no deseados. El ruido es, pues, algo objetivo, algo físico, que está ahí y tiene unas fuerzas que lo producen $y$, al mismo tiempo, es un fenómeno subjetivo que genera sensaciones de rechazo en un oyente. Tratando de objetivar los elementos que integran el ruido se pueden distinguir estos tres: la causa u objeto productor del sonido, la transmisión de la vibración, y el efecto o reacción fisiológica y psicológica que se produce en la audición (García y Javier, 2003).

En ambos casos, las teorías de contaminación acústica, sostienen que debemos proteger la salud de las personas y del medio ambiente, con 
ellos coincidimos, y recomendamos a través de este estudio, que se promueva en todos los estudiantes escolares y universitarios para desarrollar conciencia sobre la responsabilidad social en contaminación acústica y el cuidado del medio ambiente.

\section{REFERENCIAS BIBLIOGRÁFICAS}

Córdova, I. (2018). Instrumentos de Investigación. Lima, Perú: Editorial San Marcos E.I.R.L., editor.

Ecologistas en acción. (2004). La Contaminación A c ú $s$ t i ca. O b t e $\mathrm{n}$ i do d e : https://www.ecologistasenaccion.org/article 5350.html

Fact s (2017). Noise in Figures. Obtenido de: https://osha.europa.eu/en/tools-andpublications/publications/factsheets/67?set language $=\mathrm{es}$

Fernández, L. (2000). Conceptos Físicos de las Ondas Sonoras. Física y Sociedad, revista del Colegio Oficial de Físicos. No 11. O b t e n i d o d e : https://www.cofis.es/pdf/fys/fys11/fys11_co mpleto.pdf

García, B. \& Javier F. (2003). La Contaminación Acústica en nuestras ciudades. Obtenido De: https://www.fundacionbancarialacaixa.org/d
eployedfiles/obrasocial/Estaticos/pdf/Estudi os_sociales/es12_esp.pdf

Hernández, R., Fernández, C.\& Baptista, M. (2014). Metodología de la investigación. México D.F. Sexta Edición. Editorial McGraw-Hill.

Nel, L. (2010). Metodología de la Investigación, Estadística Aplicada en la Investigación. Lima, Perú. Empresa Editora Macro E.I.R.L.

Ruiz, J. (1999). Metodología de la Investigación Cualitativa. Universidad de Deusto. Bilbao.

Sánchez, H. y Reyes, C. (2017). Metodología \& Diseños en la Investigación Científica. Lima, Perú. Editorial Business Support Aneth S.R.L.

Waste (2017). Contaminación Acústica y Salud. O b t e n i d o d e : http://waste.ideal.es/acustica.htm

Correo electrónico: lopez2410@yahoo.es

Revisión de pares:

Recibido: 12/09/2019

Aceptado: 18/12/2019 